\title{
Modélisation de l'indentation des structures sandwichs à peaux métalliques
}

\author{
Y. Aminanda ${ }^{1}$, B. Castanién ${ }^{1, a}$, J.-J. Barrau ${ }^{2}$ et P. Thevenet ${ }^{3}$ \\ 1 IGMT/LGMT, LMS Supaéro, BP 4032, 31055 Toulouse Cedex 4, France \\ 2 IGMT/LGMT, Bât. 3PN, Université Paul Sabatier, 118 route de Narbonne, 31062 Toulouse, France \\ 3 EADS CCR, 12 rue Pasteur, BP 76, 92152 Suresnes Cedex, France
}

Reçu le 10 juillet 2003, accepté le 10 novembre 2004

\begin{abstract}
Résumé - Une modélisation de l'indentation des structures sandwichs à peaux métallique et âme en nid d'abeille est présentée dans cette étude. Cette recherche s'effectue dans le cadre plus général de l'étude de l'impact basse vitesse/basse énergie sur les structures sandwichs en considérant que l'équivalence statique/dynamique est valable pour les structures et gammes d'impact étudiés. Le point-clef de la modélisation est de pouvoir représenter de manière simple et robuste numériquement le comportement nonlinéaire du nid d'abeille à l'écrasement. Afin de décrire de manière plus explicite que dans la littérature les plissements du nid d'abeille constatés expérimentalement, une étude phénoménologique est d'abord réalisée. Il s'avère que le comportement à l'écrasement est piloté par les arêtes verticales de la structure cellulaire. Un modèle analytique simple d'indentation du nid d'abeille seul peut alors être proposé. Il est in fine basé uniquement sur une loi de comportement obtenue par un essai de compression uniforme. Ce modèle permet une très bonne simulation de l'indentation des structures nid d'abeille seules pour diverses géométries d'indenteur. Dans un deuxième temps, l'indentation des structures sandwichs à peaux métalliques et âme nid d'abeille est étudiée. Un modèle éléments finis est proposé dans lequel le nid d'abeille est modélisé par une grille de ressorts non-linéaires verticaux. Les ressorts représentent les arêtes verticales du nid d'abeille. En utilisant la loi expérimentale obtenue lors de l'essai de compression uniforme, il est alors aussi possible de corréler correctement les lois de contact d'essais d'indentation statique de structure sandwich à nid d'abeille Nomex ${ }^{\mathrm{TM}}$ et à peaux métalliques. Les résultats obtenus par cette approche pourront a priori être étendus au cas des structures sandwichs à peaux composites et à la détermination de la résistance après impact de ces structures.
\end{abstract}

Mots clés : Structures sandwich / indentation statique / impact basse vitesse / basse énergie / éléments finis non-linéaires

\begin{abstract}
Modelling of the static indentation of honeycomb core sandwichs with metallic skins. In this research, the study and modelling of the low velocity/low energy impact on sandwich structures with metallic skins is carried out by considering that the static/dynamic equivalence is valid for the structures and ranges of impact studied. A phenomenological study of the behaviour of honeycomb subjected to crushing is completed first, making it possible to propose a simple analytical model based on a uniform compression test. This model is used to create a good simulation of honeycomb structures in indentation for various indentor geometries. A finite element model can then be made based on nonlinear springs and which correlates with the contact laws of static indentation tests of metal-skins Nomex ${ }^{\mathrm{TM}}$ honeycomb sandwich structures.
\end{abstract}

Key words: Sandwich structures / low velocity / low energy impact / finite element nonlinear analysis / static indentation

\section{Introduction}

Les structures sandwich présentent des grandes raideurs de flexion spécifiques et des tenues au flambage

a Auteur correspondant : bruno.castanie@supaero.fr élevées qui sont des critères de premières importance dans le domaine aéronautique. Pourtant, leur domaine d'application reste limité aux structures secondaires tels que des ailerons ou des planchers. De fait, une des principales limitations à l'extension de leur utilisation est due au 
manque de connaissance de leur comportement à l'impact (Abrate [1]). Pourtant, il est fréquent qu'en service ces structures lorsque qu'elles sont apparentes soient impactées par des projections de cailloux ou des débris lors de manœuvres de roulage voire par des oiseaux au décollage. Elles sont aussi susceptibles d'être détériorées par des chutes d'outils lors d'opérations de maintenance (Guedra-Degeorges [2]; Razi [3]). Le problème se pose alors à l'avionneur de pouvoir dire à la compagnie, dans des délais réduits, si un impact doit être réparé ou non. Si des outils existent (Guedra-Degeorges [2]), ceux-ci s'avèrent extrêmement coûteux en temps de calcul et dans l'état actuel ne représentent qu'imparfaitement les phénomènes physiques mis en jeu. L'étude présentée dans cet article s'inscrit dans ce contexte général de recherche et se limite à la modélisation de l'impact et des dommages causés sur des sandwichs à âme nida Nomex ${ }^{\mathrm{TM}}$ et peaux métalliques pour s'affranchir dans un premier temps des modes complexes de dégradation des peaux stratifiées.

Le type d'impact concerné par cette étude est du type basse vitesse/basse énergie. Des essais réalisés au laboratoire ainsi qu'une majorité d'auteurs s'accorde à dire que dans ce domaine d'impact et pour ce type de sandwichs aéronautiques dont l'épaisseur de peau n'excède pas 2,5 mm, il existe une équivalence entre essai dynamique et indentation statique (Ferry [4]; Williamson [5]; Herup [6]). L'intérêt d'utiliser alors des essais statiques est une mise en ouvre plus simple et une dispersion inférieure des résultats. De plus la modélisation des phénomènes peut se faire de manière plus aisée avec un logiciel de calcul éléments finis explicite du commerce. Par conséquent, dans la suite de cet article, seule l'indentation statique des structures sandwichs sera considérée.

L'indentation des structures sandwichs peut être déterminée par simple recalage de lois de contact de type Hertz [1]. Des modèles analytiques à partir d'études de poutres ou plaques sur fondations élastiques ont aussi été développés par un faible nombre d'auteurs. Les approches les plus anciennes s'appuient sur la théorie de l'élasticité donc leur application est très limité en pratique [1]. Swanson [7]; Soden [8] et Olsson [9] proposent des modèles avec fondation parfaitement plastique censée représenter l'âme. La cinématique est linéaire dans le modèle de Soden [8] et en grands déplacements pour Olsson [9]. Toutefois, ces modèles présentent un intérêt restreint dans le cadre de notre étude car lors de l'indentation les peaux métalliques plastifient et l'âme en nid d'abeille se détériore par plissement de l'âme (Bernard [10]; Tsotis [11]). Ce dernier mode de dégradation est présent dès les plus faibles énergies d'impact et ses connaissance et compréhension sont le point clef de la modélisation de l'impact ou de l'indentation sur structure sandwich. C'est un phénomène mécanique complexe caractérisé par l'apparition de plis et de ruptures diverses dans la structure hexagonale. Ce phénomène est connu pour ses capacités d'absorption d'énergie et des modèles semi-empiriques le décrivant du point de vue global existent dans le cas de nids d'abeilles en alliages d'aluminium (Mc Farland [12]; Wierzbicki [13]). Par ailleurs, la forme et la répartition des plis obéissent à des lois géométriques (Enboa [14]). Pour le Nomex ${ }^{\mathrm{TM}}$, la micromécanique est complexe et des ruptures de résine en surface, des décollements locaux et des déchirures sont observées (Tsotis [11]; Aminanda [15]). De ce fait, il semble peu probable qu'une modélisation éléments finis soit apte à simuler l'ensemble de ces phénomènes locaux dans un contexte industriel (Aminanda [15]). D'une manière générale, le mécanisme de formation des plis n'est pas ou peu explicité alors que le phénomène est commun aux structures cellulaires. On l'observe en particulier au microscope dans les cellules de mousse (Thomson [16]). Les modélisations de ce phénomène reposent en général sur une loi matériau équivalente qui peut être de type parfaitement plastique (Besant [17]) ou en discrétisant la loi d'écrasement de pains de nids d'abeille (Zheng [18]; Minguet [19]). Dans ce cadre, Horrigan [20] utilise une loi d'endommagement isotropique continue mais cette modélisation est restreinte aux faibles indentations car la continuité du modèle de dégradation n'est pas représentative de l'état réel d'endommagement de l'âme. La connaissance de la zone endommagée conditionne la détermination de la résistance après impact de la structure et pour palier à l'absence de modèle efficace, Lacy [21] utilise les résultats d'une campagne d'essai pour modéliser l'étendu du dommage.

Afin de hiérarchiser les difficultés de cette problématique, la démarche retenue est progressive. Dans un premier temps, une étude phénoménologique de l'indentation des structures nid d'abeille seules est effectuée. L'observation des mécanismes mis en jeu lors de l'écrasement permet alors de proposer un modèle analytique simple qui permet de corréler la loi de contact expérimentale d'indentation des nids d'abeille Nomex ${ }^{\mathrm{TM}}$. Dans un deuxième temps, cette approche sera reprise pour modéliser l'indentation des structures sandwichs à âme nid d'abeille Nomex ${ }^{\mathrm{TM}}$ et peaux métalliques.

\section{Indentation statique des nids d'abeille seuls}

\section{1 Étude phénoménologique}

L'étude bibliographique a montré que les structures cellulaires (mousses, nids d'abeille) ont toutes globalement le même comportement en compression qui se manifeste par la présence de plissements. Aussi, il semble possible qu'un raisonnement qualitatif par analogie puisse être mené. En effet, la difficulté pratique pour mettre en évidence les mécanismes de formation des plissements tient à la petite taille des cellules des nids d'abeille utilisés dans les structures aéronautiques, à leur aspect fermé et à la difficulté d'observation qui en résulte. Aussi, un nid d'abeille de grandes dimensions a été réalisé manuellement au laboratoire à l'aide de simple papier dessin épais. Ce nid d'abeille est collé sur une ou deux peaux en plexiglas. La structure obtenue est ensuite posée sur un bloc en plastique avec des hublots en plexiglas et une caméra CCD est installée ce qui permet de filmer la compression 


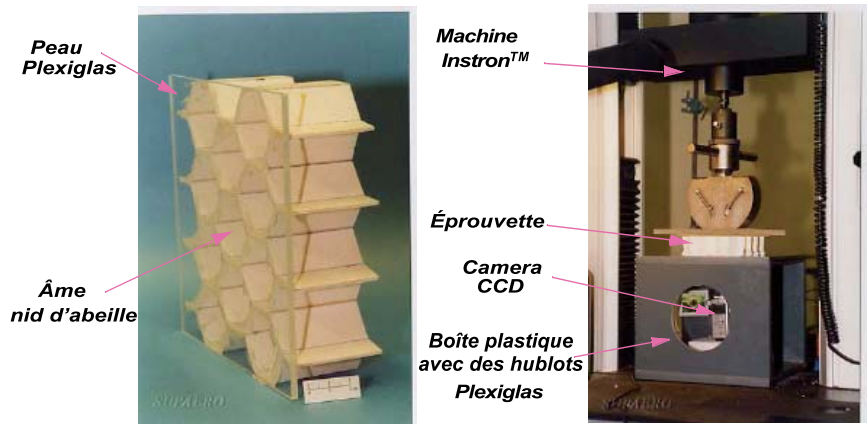

Fig. 1. Éprouvette en papier Canson et montage d'essais.

de l'éprouvette (voir Fig. 1). Parallèlement, des essais de compression uniforme sur des nids d'abeille en aluminium et en Nomex ${ }^{\mathrm{TM}}$ sont réalisés. De la même manière, une ou deux peaux en alliage d'aluminium peuvent être collées sur ces éprouvettes (voir Tab. 1). L'essai se fait en pilotant le déplacement avec une vitesse de $0.5 \mathrm{~mm} \cdot \mathrm{min}^{-1}$.

Le mécanisme de formation observé pour une éprouvette à une seule peau est le suivant (voir Fig. 2) :

- Première étape : Apparition d'un flambage localisé sur les faces des hexagones côté non collé. Les arêtes verticales de la cellule restent droites. Les conditions aux limites de type simplement appuyé qui autorisent une rotation locale de la section semblent à l'origine de l'initiation du phénomène.

- Deuxième étape : Apparition du premier plissement et début de formation du second. Dans cette étape, la déformée de la première étape augmente progressivement jusqu'à ce que l'arête verticale commence à se déformer et à être enfoncée. À ce moment, le pli se forme soit à l'intérieur soit à l'extérieur de la ligne hexagonale de la cellule initiale. L'alternance est pilotée par les effets présents au niveau de l'arête verticale qui est la jonction de trois branches appartenant à 3 cellules différentes. On observe à cet endroit des interpénétrations, des déchirures locales et des décollements locaux. Simultanément, le fait que l'arête ne soit plus droite initie la formation du deuxième pli.

- Troisième étape : Le premier pli s'aplatit complètement alors que le deuxième pli se forme et commence à se propager au troisième.

- Étapes suivantes : Même mécanisme et propagation du plissement.

Ce mécanisme de déformation est exactement le même sur toutes les éprouvettes réalisées en nida Canson. La hauteur et la densité (donc l'épaisseur) du nida n'ont pas d'influence sur le mécanisme de la déformation. Par contre, la longueur du plissement est influencée par l'épaisseur de la plaque constituant une face de la cellule. La forme des plis est différente suivant les matériaux : angles vifs pour le Nomex ${ }^{\mathrm{TM}}$ et arrondis pour l'alliage d'aluminium et le papier. Les déformées finales obtenues sont visibles figure 3 . Lors des essais de compression uniforme avec deux peaux, le plissement se produit à une hauteur aléatoire de l'éprouvette puis en général la partie haute glisse horizontalement sur la partie basse (Fig. 4).
Ce comportement n'est pas retrouvé lors des impacts sur structures sandwichs puisque le crush se produit toujours à proximité de la face impactée et d'après les essais précédents est probablement initié par des rotations locales.

La loi de comportement force/écrasement est similaire quel que soit le type de nid d'abeille (Figs. 5 et 6 ) pour une ou deux peaux. Les courbes obtenues sont bien sûr similaires à celles présentées dans la littérature. On retrouve une première montée élastique où la perte de rigidité causée par le flambage local des parois des cellules n'est pas sensible. Le passage par le maximum suivi d'une chute brutale de la raideur correspond au flambage des arêtes verticales des cellules hexagonales. La brutalité de l'effondrement varie suivant le matériau et les conditions aux limites. Le Nomex ${ }^{\mathrm{TM}}$ ayant un comportement élastique fragile, les plis formés offrent une faible résistance résiduelle alors que des rotules plastiques se forment dans le cas de l'alliage d'aluminium. La charge maximale est toujours supérieure dans le cas d'essais avec deux peaux. Il semble que l'analogie avec le flambage classique puisse être proposée puisque d'une manière générale des conditions aux limites de type encastré génèrent des charges de flambage supérieures. Une zone plateau est observée qui peut présenter une légère remontée et qui est présentée comme correspondant à la « densification »du nida. En fait les plis supérieurs s'écrasent et se propagent progressivement à effort constant dans l'épaisseur du nid d'abeille. Cette partie de l'étude expérimentale a montré l'importance qu'ont les arêtes verticales dans la transmission des efforts alors que le flambage précoce des parois fait que celles-ci n'ont qu'un rôle marginal. De ce fait, des essais complémentaires ont été réalisés sur des éprouvettes en feuille d'aluminium d'épaisseur $0,5 \mathrm{~mm}$ reprenant le motif de la cellule hexagonale au niveau d'une arête verticale. Qualitativement, les courbes d'écrasement, le motif de pli et les modes de ruptures locaux obtenus sont identiques (Fig. 7). Par ailleurs, un comportement analogue en compression peut être aussi observé dans l'écrasement de composants cruciformes des planchers d'hélicoptères (Kinderwater [22]).

Cette étude expérimentale conduit à postuler que d'un point vue structural, à l'écrasement, le nid d'abeille se comporte comme une juxtaposition d'arêtes verticales. La loi d'écrasement de ces arêtes peut alors être obtenue par compression uniforme d'un bloc de nid d'abeille dont les bords sont soigneusement découpés et collés sur une seule peau (voir Fig. 8). Le bloc comporte 100 cellules et 240 arêtes. En compression uniforme, chaque arête a un comportement identique donc la loi de comportement d'une arête est celle obtenue par l'essai divisé par 240. À partir de ces constatations un modèle analytique simple a pu être développé pour l'indentation de nids d'abeilles seuls.

\subsection{Modèle analytique et validation expérimentale}

L'objectif est ici de valider les conclusions faites suites aux observations expérimentales en particulier sur le comportement indépendant des arêtes en compression. 

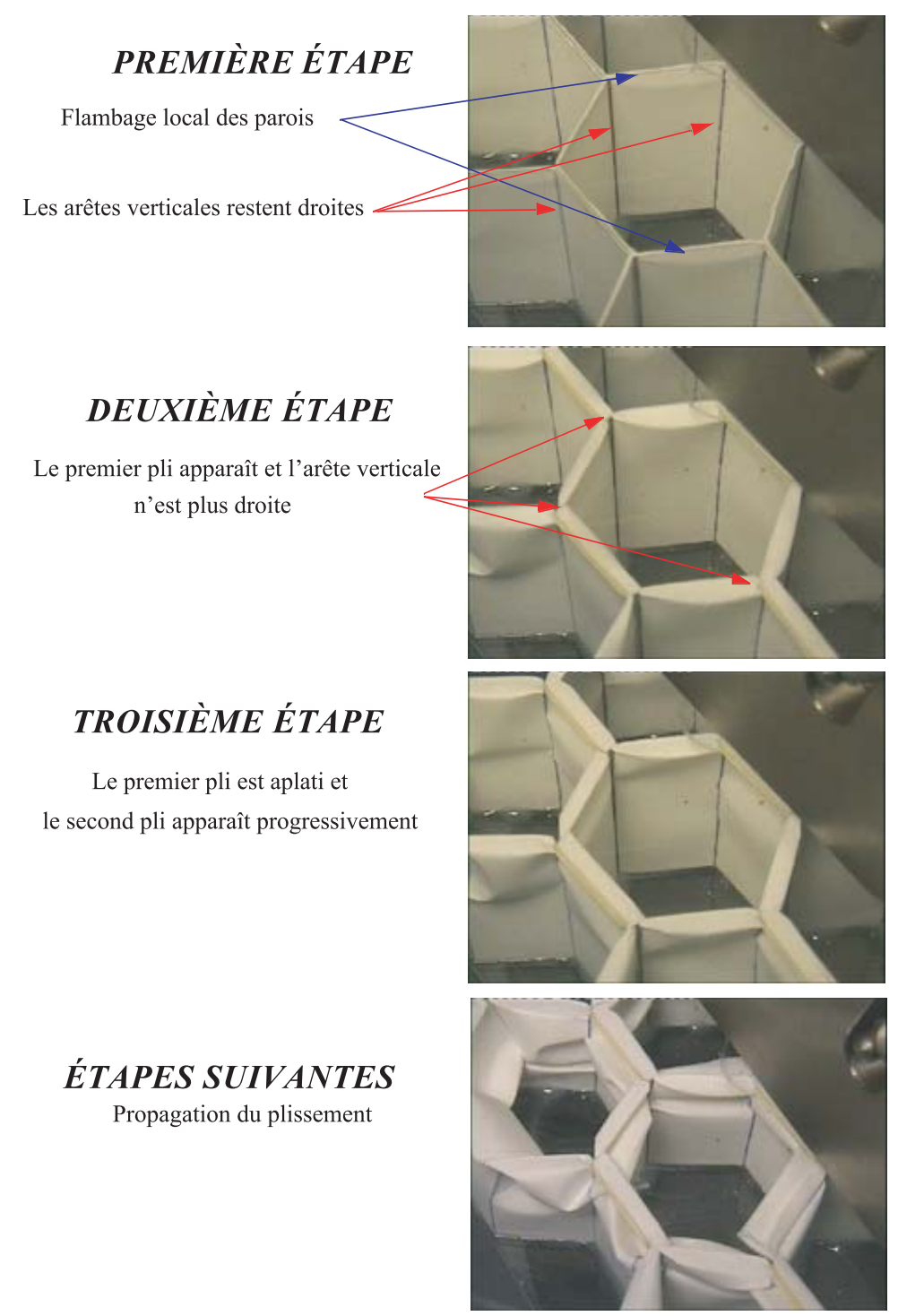

Fig. 2. Mécanisme de plissement du nid d'abeille pour une éprouvette avec une seule peau.

Tableau 1. Description des éprouvettes nid d'abeille.

\begin{tabular}{cccc}
\hline Materiaux & Papier & Nomex $^{\mathrm{TM}}$ & Alu \\
\hline Taille cellule (mm) & 35 & 5 & 6 \\
Nombre de cellules & 11 & 36 & 10 \\
Dimensions (mm) & $140 \times 140$ & $35 \times 35$ & $25 \times 25$ \\
Hauteur (mm) & 45 et 22,5 & 15 & 45 et 15 \\
Épaisseur des parois & 0,58 et 0,34 & & 0,12 \\
Nombre de peaux & 1 et 2 & 1 et 2 & 1 et 2 \\
Nombre d'éprouvettes & 8 & 2 & 4 \\
Nombre total d'éprouvettes & 8 & 4 & 4 \\
\hline
\end{tabular}

Un modèle analytique simple doit permettre de retrouver le comportement mécanique du nid d'abeille seul indenté par des indenteurs métalliques de divers rayons. Le modèle repose sur deux principales hypothèses :

1) Le chargement est repris uniquement par les arêtes verticales de la structure hexagonale.

2) Le contact indenteur/nid d'abeille est supposé parfait et donc la déformée du nid d'abeille pendant l'écrasement correspond à la forme de l'impacteur enfoncé.

La démarche de calcul est la suivante :

1) Calcul du rayon polaire des arêtes du nida $r_{i}$. L'impacteur est supposé être au centre de la première cellule indentée. 


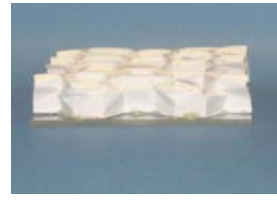

Paper

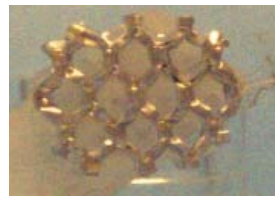

Aluminium

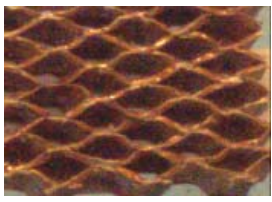

Nomex ${ }^{T M}$
Fig. 3. Déformées finales pour les éprouvettes « une peau ».

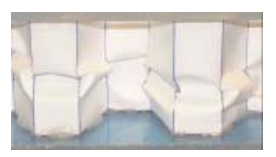

Paper

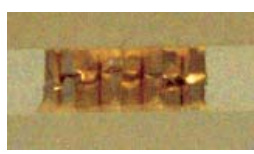

Aluminium

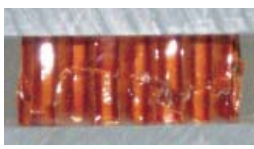

Nomex ${ }^{T M}$
Fig. 4. Déformées finales pour les éprouvettes « deux peaux ».

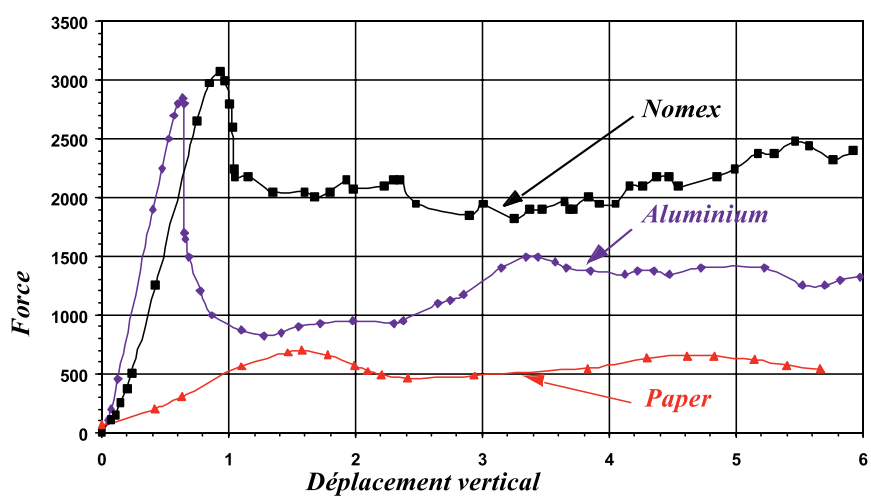

Fig. 5. Courbes force-déplacement pour éprouvettes avec une seule peau (le déplacement en abscisse est celui fourni par la machine).

2) Calcul du rayon de la surface endommagée $r_{0}$ lorsque l'impacteur s'est enfoncé d'une profondeur $z_{0}$. En notant $R$ le rayon de l'impacteur, on a : $r_{0}=$ $\sqrt{R^{2}-\left(R^{2}-z_{0}^{2}\right)}$

3) Calcul de l'écrasement de chaque arête $i$ qui se trouve à l'intérieur de la surface endommagée $\left(r_{i}<r_{0}\right): z_{i}=$ $\sqrt{R^{2}-r_{i}^{2}}-R+z_{0}$.

4) Calcul de la réaction de chaque arête $F_{i}$ à partir de l'écrasement $z_{i}$ à l'aide de la courbe de compression expérimentale discrétisée par une série de polynôme (et divisée par le nombre d'arêtes, Fig. 8).

5) La force d'indentation est $F=\sum_{i} F_{i}$.

Le modèle a été implanté sur un tableur (Excel) et la courbe force/déplacement est obtenue en incrémentant progressivement l'enfoncement de l'indenteur $z_{0}$.

Pour valider ce modèle, des essais sur du nid d'abeille Nomex ${ }^{\text {TM }}$ HRH78, 1/8-3 de 48 kg.m ${ }^{-3}$ d'épaisseur 15 mm sont effectués. Il a été indenté par des indenteurs de forme sphérique de rayons respectifs 50,$25 ; 30,125 ; 21,75$; 18,06 et $16,25 \mathrm{~mm}$ (Fig. 9). Trois essais pour chaque rayon ont été menés. La dispersion observée est faible. Les courbes d'essais présentent des petites oscillations qui correspondent aux passages des charges critiques des différentes arêtes. Ces ondulations sont moins sensibles pour l'impacteur de grand rayon car les arêtes situées en

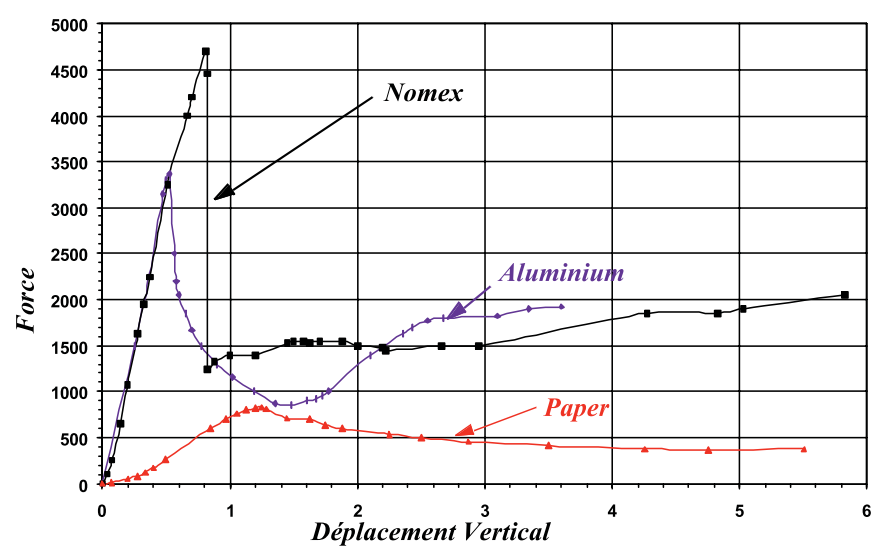

Fig. 6. Courbes force-déplacement pour éprouvettes avec deux peaux (le déplacement en abscisse est celui fourni par la machine).

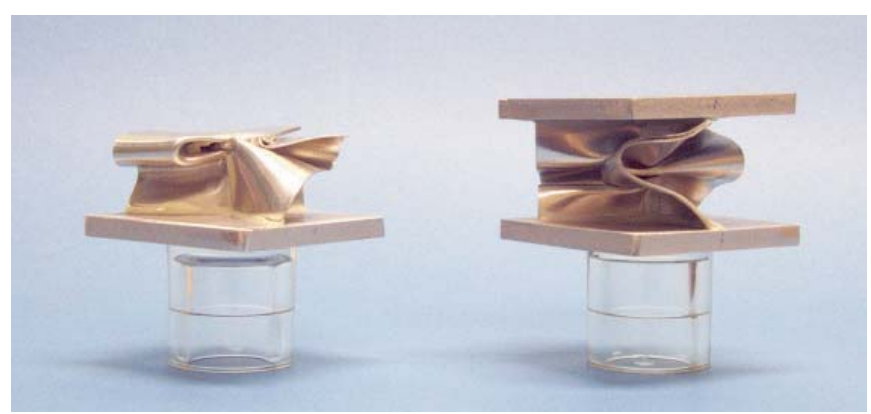

Fig. 7. Déformées finales d'éprouvettes « coin » en alliage d'aluminium.

périphérie sont écrasées presque en même temps que les arêtes centrales. De fait, on tend vers l'essai de compression uniforme quand le rayon augmente. Les essais sont stoppés quand la totalité de l'indenteur a pénétré le nid d'abeille et logiquement la force d'écrasement résultante devrait être sensiblement identique pour tous les indentateurs qui ont la même surface projetée. Or pour les rayons faibles, cette force a tendance à diminuer. L'observation des faciès d'écrasement montre que les cellules situées en périphérie ne plissent plus mais se rabattent latéralement. Par conséquent, la réaction à l'écrasement est fortement diminuée. Toutefois, ce phénomène est marginal dans le contexte de l'étude et ne remet pas en cause les développements effectués. Hormis ce phénomène de rabattement, la comparaison entre les résultats du modèle analytique et les essais est très bonne (voir Figs. 10-12). Les interprétations de l'étude phénoménologique et la démarche par analogie sont donc validées.

L'étude phénoménologique a permis d'expliciter le mécanisme de formation des plis. Celui-ci est fortement sensible aux conditions aux limites (en fait aux rotations locales) et l'essentiel des efforts transite par les arêtes verticales. Le modèle analytique développé et sa bonne capacité à représenter le phénomène d'indentation montre que d'un point de vue structure, il va être possible de représenter la structure nid d'abeille uniquement par ses arêtes ayant une loi d'écrasement non-linéaire. Cette 


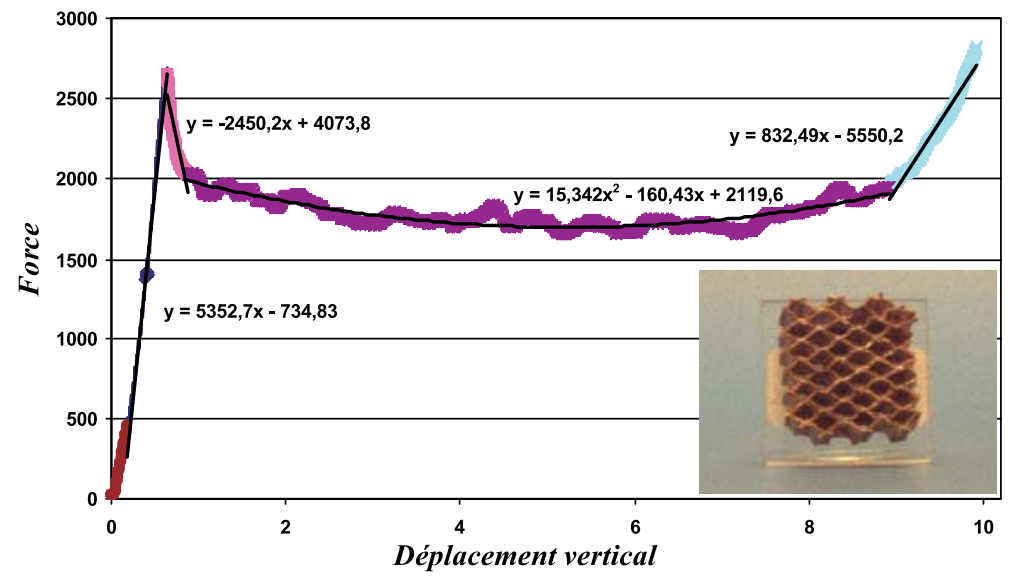

Fig. 8. Obtention de la loi de comportement des arêtes verticales (éprouvette une peau).

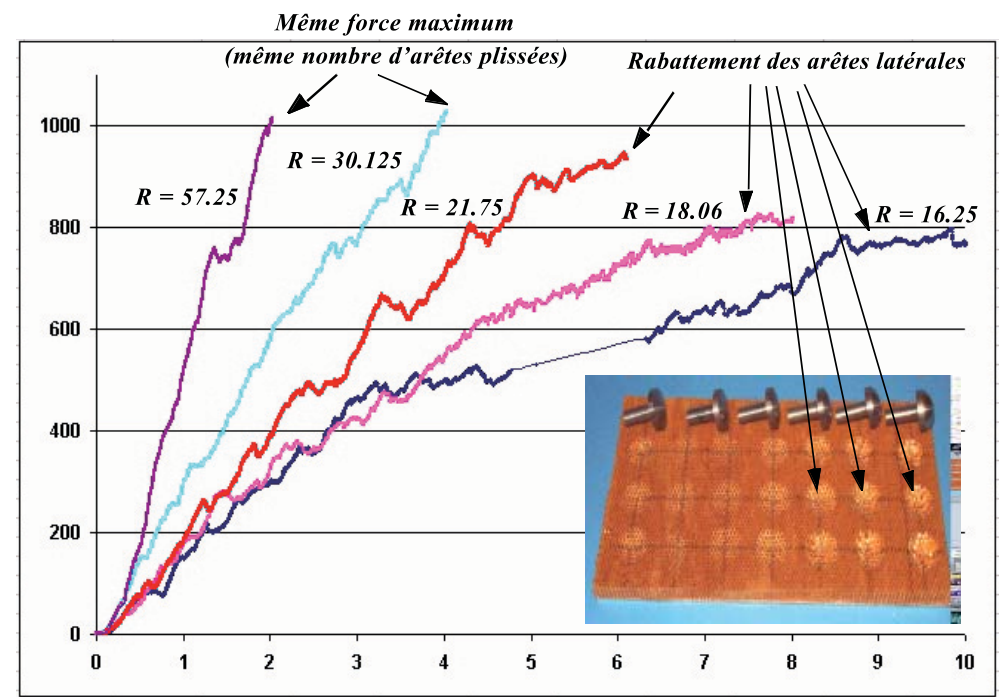

Fig. 9. Résultats d'essai d'indentation sur nida seul.

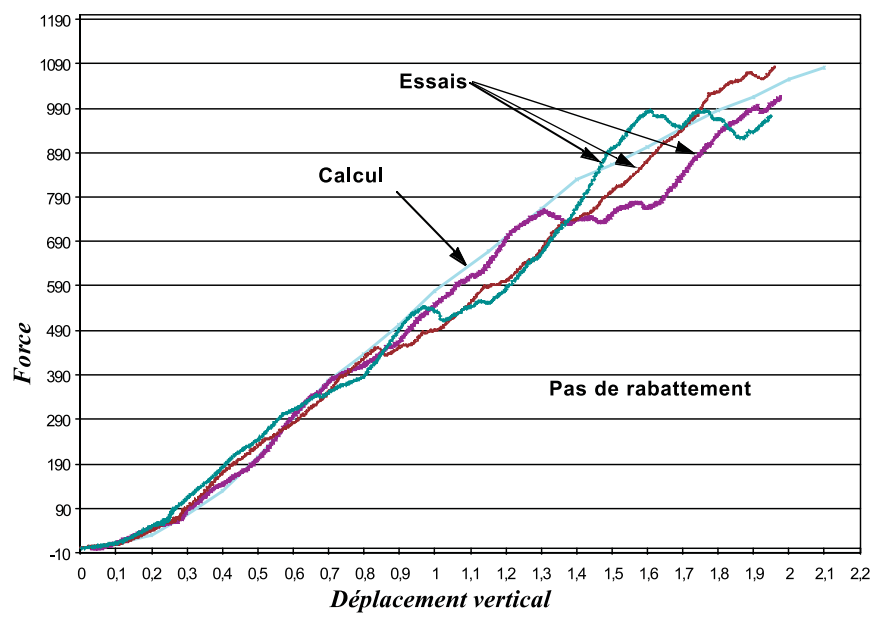

Fig. 10. Comparaison entre calcul et essais d'indentation avec indenteur sphérique $(R=57,25 \mathrm{~mm})$.

loi est obtenue expérimentalement par un essai simple de compression uniforme. Il est fort probable que cette approche soit extensible à de nombreux matériaux cellulaires mais le contexte industriel de l'étude fait que des approfondissements n'ont pu être menés dans cette voie. À présent, les conclusions de cette étude vont être reprises pour modéliser l'indentation de structures sandwichs à âme Nomex ${ }^{\mathrm{TM}}$ et peaux métalliques.

\section{Indentation des structures sandwichs à peaux métalliques}

\subsection{Description des essais}

Des essais d'indentation quasistatiques sont menés sur des structures sandwichs de taille $100 \times 100 \mathrm{~mm}$, fabriquées en utilisant le même nida Nomex ${ }^{\mathrm{TM}}$ de taille de cellule $3,2 \mathrm{~mm}$, de hauteur $15 \mathrm{~mm}$ et de densité de $48 \mathrm{~kg} \cdot \mathrm{m}^{-3}$ (HRH 78,1/8,3). Les peaux en laiton d'épaisseurs 0,1 (peau fine) et $1 \mathrm{~mm}$ (peau épaisse) sont collées avec le nida Nomex ${ }^{\mathrm{TM}}$ en utilisant un film de colle REDUX ${ }^{\mathrm{TM}} 312 / 5$. Les éprouvettes fabriquées sont 
Tableau 2. Détail des éprouvettes sandwich fabriquées.

\begin{tabular}{cccc}
\hline Matériaux des sandwichs & Épaisseur de peau & Taille de l'éprouvette & Nombre \\
\hline Peau : Laiton & $0,1 \mathrm{~mm}$ & $100 \times 100 \mathrm{~mm}$ & 9 \\
Âme : $\operatorname{Nomex}^{\mathrm{TM}}(\mathrm{HRH} 78,1 / 8,3)$ & $1 \mathrm{~mm}$ & $100 \times 100 \mathrm{~mm}$ & 9 \\
\hline
\end{tabular}

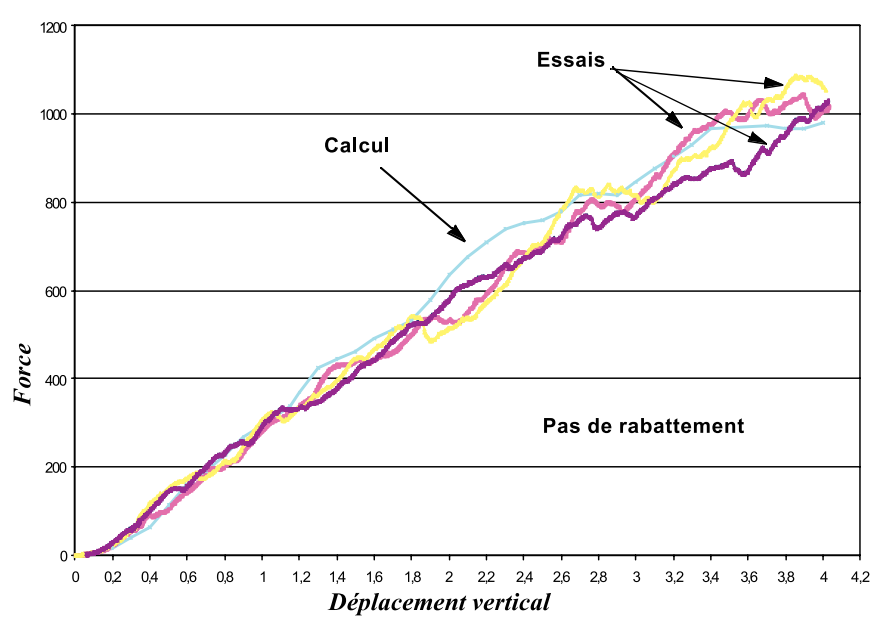

Fig. 11. Comparaison entre calcul et essais d'indentation avec indenteur sphérique $(R=30,125 \mathrm{~mm})$.

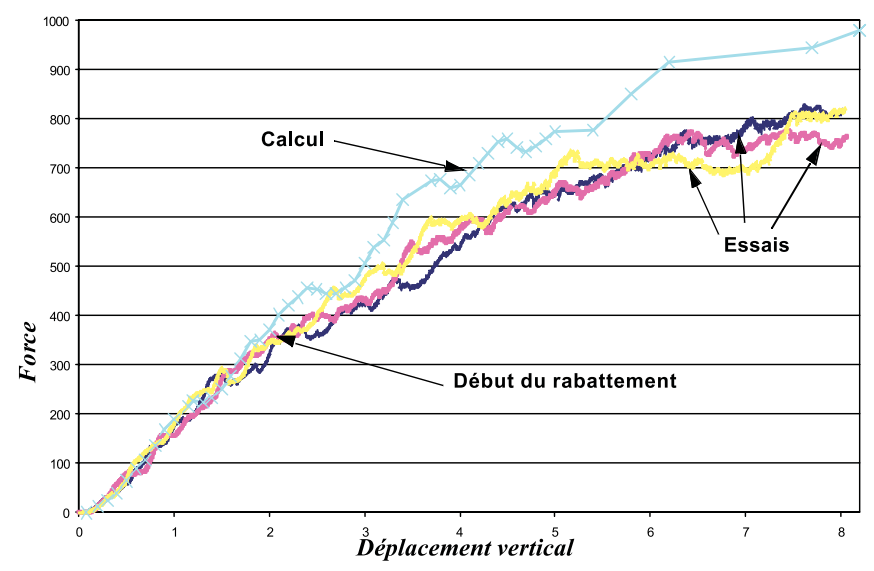

Fig. 12. Comparaison entre calcul et essais d'indentation avec indenteur sphérique $(R=18,06 \mathrm{~mm})$.

listées tableau 2. Les éprouvettes sont indentées en utilisant la même machine INSTRON ${ }^{\mathrm{TM}}$ et les mêmes indenteurs sphériques de rayons différents $(57,25 ; 31,75$ et 21,25 mm) (Fig. 13). Trois essais par type de sandwich (peau fine ou épaisse) et par indenteur sont réalisés. Une vitesse d'écrasement de $0,5 \mathrm{~mm} . \mathrm{min}^{-1}$ est utilisée pour pouvoir considérer que le chargement est quasi-statique. L'éprouvette est appuyée sur toute sa surface sur un support métallique. Les efforts sont mesurés par le capteur de la machine mais les déplacements sont mesurés par des comparateurs mécaniques placés sur l'indenteur.

La figure 14 montre l'aspect des éprouvettes avec peaux d'épaisseur $0,1 \mathrm{~mm}$ après indentation. Pour cette épaisseur de peaux, des lignes de rupture sont apparentes en fond d'empreinte pour les indenteurs de diamètre 31,75 et 21,25 mm. Pour les peaux épaisses de $1 \mathrm{~mm}$ d'épaisseur,

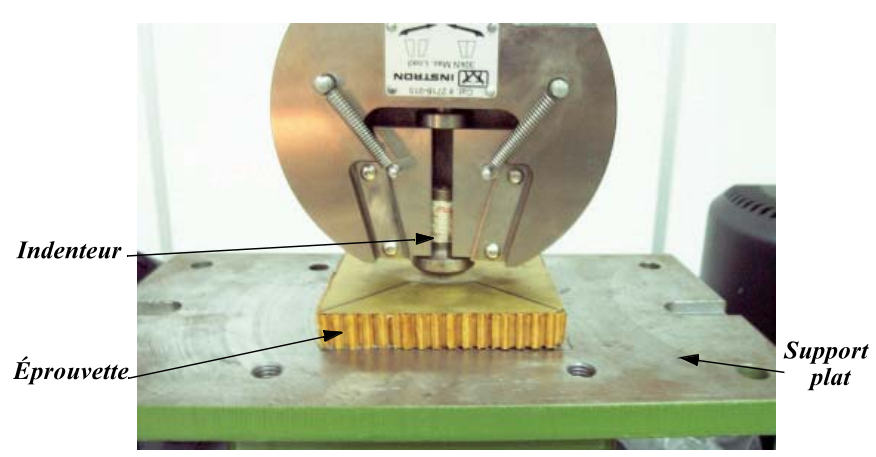

Fig. 13. Description des essais d'indentation.

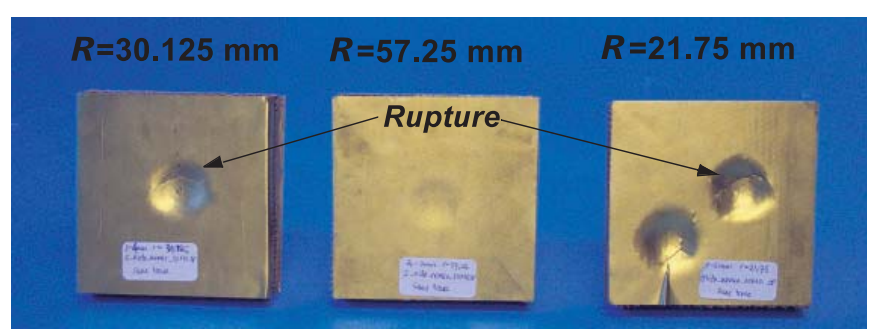

Fig. 14. Aspect final des éprouvettes sandwichs après indentation.

aucune rupture n'est apparente et seule une empreinte est visible. Les lois de contacts obtenues montrent une dispersion des résultats faibles sauf pour les charges extrêmes dans le cas des peaux de 0,1 mm d'épaisseur. Cette dernière dispersion semble due à l'apparition des fissures en fond d'empreinte. Les lois de contact expérimentales sont présentées figure 15 (peau 0,1 mm) et figure 16 (peau $1 \mathrm{~mm}$ ). Pour la peau de $0,1 \mathrm{~mm}$ l'allure des courbes d'écrasement ne présente pas de particularités notables à l'exception de petites ondulations similaires à celles observées avec nida seul. Pour la peau de $1 \mathrm{~mm}$, la rigidité est très importante au début puis plus faible par la suite. Ce changement peut qualitativement être attribué à l'effondrement de la première cellule de nid d'abeille sous l'indenteur. En effet, d'une part le changement de pente s'observe pour une valeur d'enfoncement $(0,29 \mathrm{~mm})$ qui correspond aussi au passage du « pic » obtenu aussi par essai sur nida seul, d'autre part un bruit caractéristique est entendu à ce moment. Les comportements différents observés entre peaux épaisses et peaux fines suggèrent que le mode de plissement des arêtes verticales doit différer. Il est probable que la peau fine qui possède une rigidité de flexion faible épouse presque parfaitement la forme de l'indenteur (Fig. 17a) alors que la peau épaisse du fait d'un moment quadratique de flexion 1000 fois supérieur se déforme de manière différente (Fig. 17b). Par conséquent, la déformée de flexion de la peau épaisse doit imposer des 


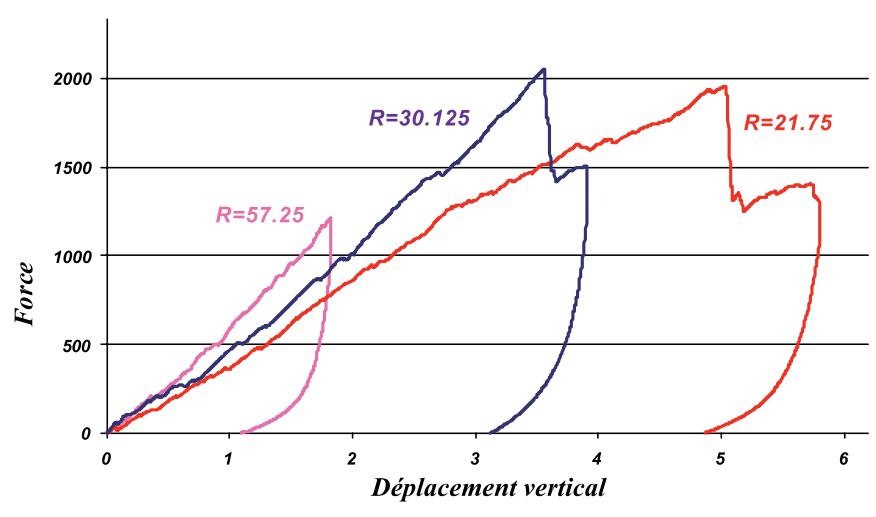

Fig. 15. Courbe force/déplacement pour sandwich peau laiton épaisseur 0,1 $\mathrm{mm}$.

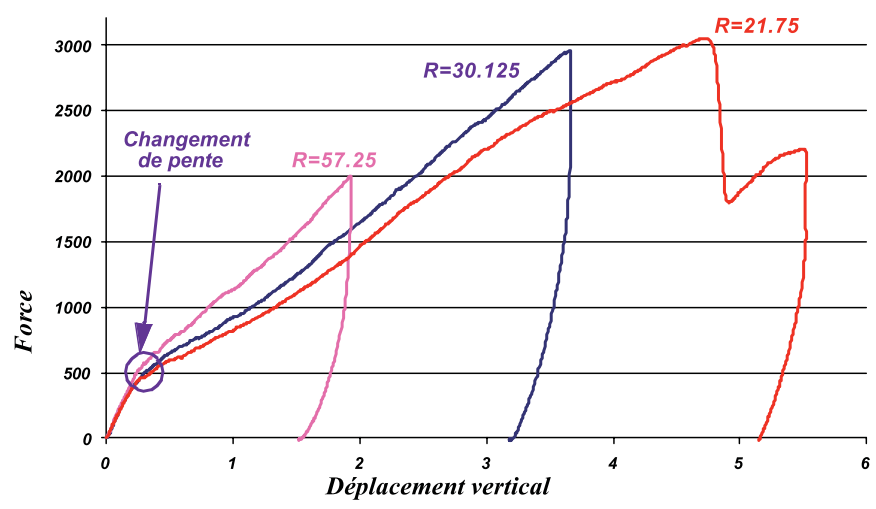

Fig. 16. Courbe force/déplacement pour sandwich peau laiton épaisseur $1 \mathrm{~mm}$.

rotations locales aux arêtes bien avant que l'indenteur soit en contact avec l'arête. Par ailleurs, dans la littérature, des observations expérimentales ont permis de montrer que les arêtes restent perpendiculaires localement à la peau [23] et donc subissent les rotations de celle-ci. Par ailleurs, les essais de compression uniforme avec une ou deux peaux ont montré la sensibilité du mécanisme de plissement aux conditions aux limites. Il est clair que la prise en compte de ce comportement devra être fait dans la modélisation proposée. Il est aussi important de noter à ce niveau que des modélisations volumiques classiques intégrant la loi de compression non-linéaire ne peuvent pas rendre compte de cette sensibilité aux conditions aux limites en rotation.

\subsection{Modélisation éléments finis des sandwichs à peau métallique fine}

Un modèle éléments finis non-linéaire est élaboré à partir des constations phénoménologiques effectuées dans la première partie de l'étude. Le logiciel utilisé est le code SAMCEF (SAMTECH GROUP). Une grille de ressorts verticaux placés à chaque emplacement géométrique des arêtes du nid d'abeille modélise celui-ci (Fig. 18). La loi d'écrasement de ces ressorts est non-linéaire et provient d'un essai de compression uniforme sur un pain de nid

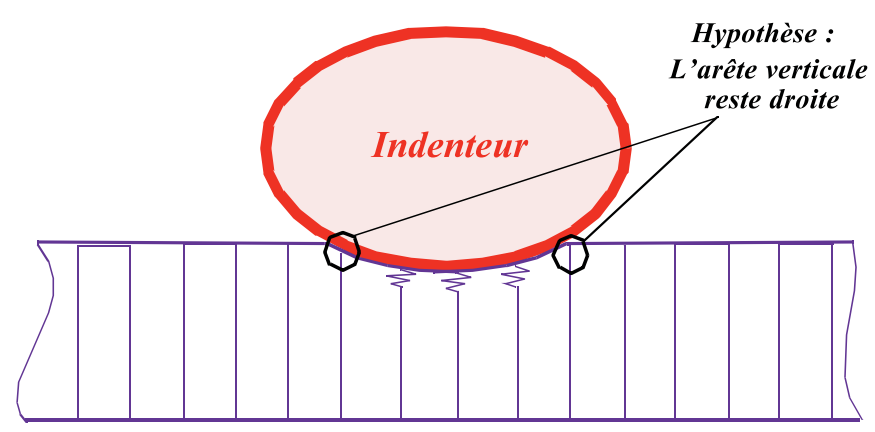

(a) : Sandwich avec peaux fines.

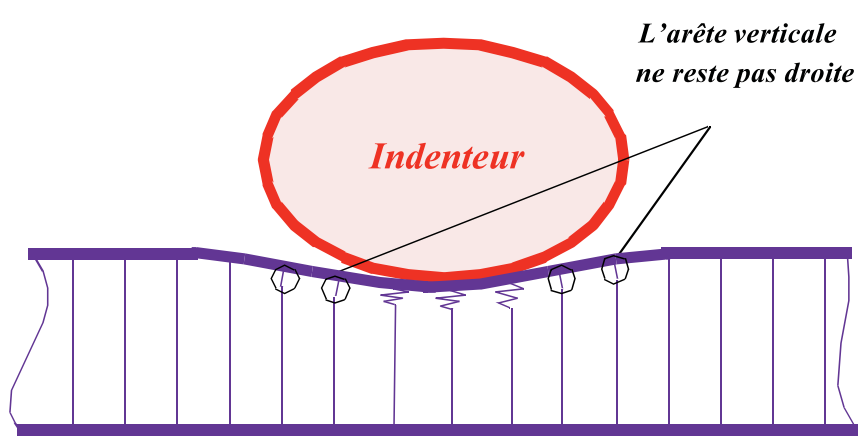

(b) : Sandwich avec peaux épaisses.

Fig. 17. Explication physique de la différence de comportement entre sandwichs à peaux fines et peaux épaisses.

d'abeille à 240 arêtes avec deux peaux. En effet, la similitude est évidente et les conditions aux limites sur les arêtes sont plus proches. Il est ainsi fait implicitement l'hypothèse que la rotation locale de la peau lors de l'indentation dans le cas des peaux fines coïncide ou précède de peu le plissement et par conséquent affecte peu la loi obtenue expérimentalement en compression uniforme (Fig. 17a). Les peaux métalliques sont modélisées par des éléments de plaques de Mindlin et la loi élastoplastique du laiton est celle obtenue à la suite d'un essai de traction classique. Un maillage fin est utilisé au droit de l'indenteur pour générer une loi de contact sans rupture de pente. Enfin, seul un quart de plaque est modélisé du fait des symétries matérielles. La simulation numérique est limitée à $2 \mathrm{~mm}$ d'indentation ce qui correspond à un seuil de détectabilité dit BVID (Barely Visible Impact Damage).

Le modèle est ensuite comparé aux essais réalisés et une bonne comparaison essai/théorie est obtenue pour les trois diamètres d'indenteur (Fig. 19) ce qui valide a posteriori l'intérêt de l'étude phénoménologique et l'hypothèse que la loi expérimentale obtenue en compression uniforme reste valide.

\subsection{Modélisation éléments finis des sandwichs à peau métallique épaisse}

Des essais numériques ont montré que l'utilisation directe du modèle précédent donnait une corrélation des 


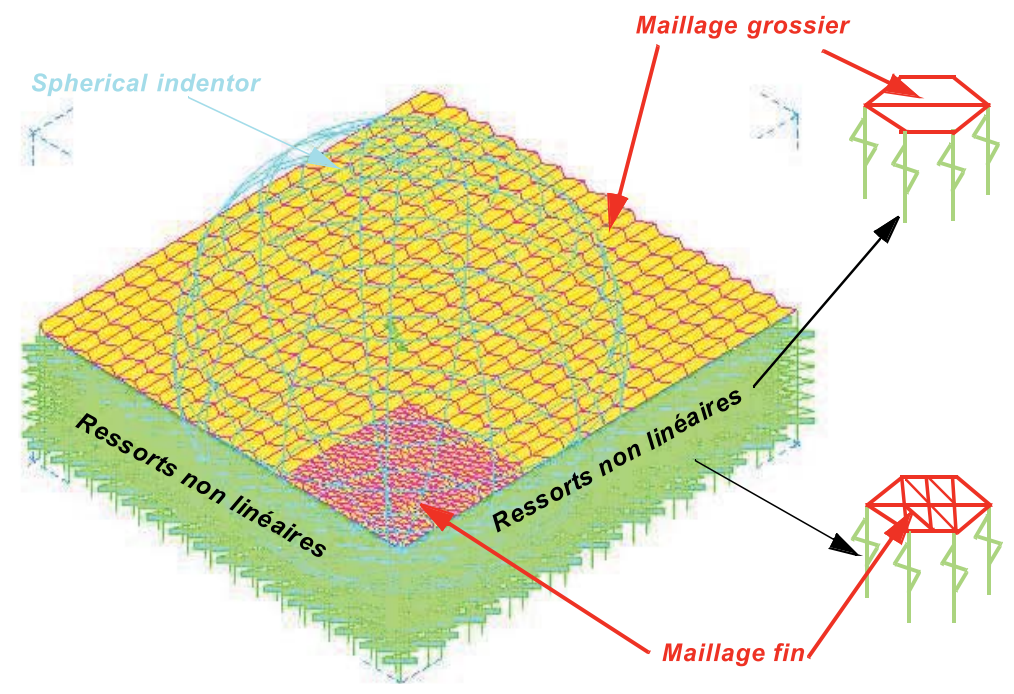

Fig. 18. Modélisation éléments finis du problème d'indentation sur sandwich à peaux métalliques fines.

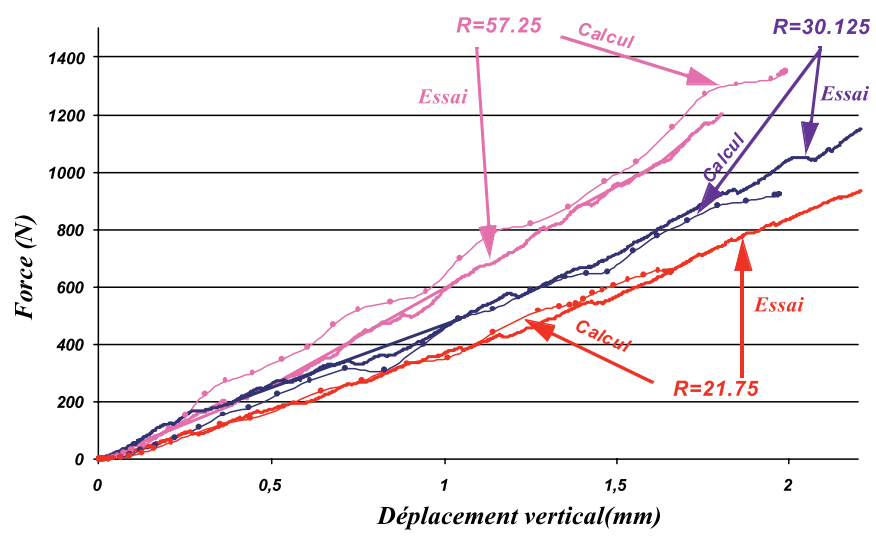

Fig. 19. Corrélation essai/modèle numérique dans le cas sandwich à peaux métalliques fines.

essais peu satisfaisante. En fait, comme identifié lors de l'étude expérimentale, une des insuffisances de la modélisation précédente est une mauvaise représentation du phénomène de rotation locale imposée par la peau épaisse. Aussi, la modélisation retenue de ce phénomène consiste à conserver la loi « avec pic» pour les arêtes appartenant à la première cellule indentée et à imposer une loi « sans pic» pour toutes les autres (Fig. 20). Par ailleurs, une forte triaxialité des contraintes au droit de l'indenteur impose un maillage avec plusieurs éléments dans l'épaisseur. Une étude de sensibilité au maillage a été effectuée et la convergence a été obtenue pour 5 éléments dans l'épaisseur et 36 éléments par cellule. La comparaison des essais avec cette modélisation s'avère globalement satisfaisante (Fig. 21). Il est toutefois notable que la première partie de l'essai jusqu'au changement de pente est assez bien représentée par le modèle mais que au-delà la représentation est moins pertinente. Cela semble dû à une représentation du comportement des arêtes du nid d'abeille qui ne prend pas en compte le caractère progressif du passage « loi avec pic » à « loi sans pic ».
La présence de deux modèles montre des différences en termes de comportement de la réponse à l'indentation entre les sandwichs à peaux fines et peaux épaisses. Chaque modèle présente certains points faibles et il a été nécessaire de développer un modèle unique permettant de décrire l'ensemble des phénomènes mécaniques.

\subsection{Modélisation de l'indentation par un modèle unifié}

Des éléments de plaque de Mindlin ont été choisis pour modéliser la peau car d'une part leur coût en terme de C.P.U. est plus faible que les éléments volumiques et d'autre part leur formulation comprend des termes de rotation aux nouds. De fait, la modélisation initiale (Fig. 18) est globalement reprise. Les deux phénomènes physiques à représenter sont d'une part la triaxialité des contraintes au droit de l'indenteur et d'autre part le phénomène de flexion de la peau épaisse qui modifie la réponse des arêtes verticales du nid d'abeille.

Des essais numériques ont montré que la triaxialité des contraintes au droit de l'indenteur conduit à retarder la plastification de la première cellule uniquement. Aussi, il a été proposé de modifier la loi de comportement de cette première cellule en augmentant artificiellement le seuil de plastification (Fig. 22). Pour fixer un nouveau seuil représentatif, un essai d'indentation sur support plat d'une poutre sandwich à peaux laiton $1 \mathrm{~mm}$ a été réalisé. L'indenteur est cylindrique de diamètre $5 \mathrm{~mm}$ ce qui permet de s'affranchir des effets de Poisson. Une modélisation spécifique reprenant les principes développés dans cette étude est effectuée et le seuil de plasticité des éléments au droit de l'indenteur est augmenté numériquement jusqu'à l'obtention d'une bonne corrélation essais/calculs (Fig. 23).

Le deuxième axe d'amélioration est l'utilisation d'une loi unique de détérioration des arêtes verticales. Pour cela, un élément spécifique au logiciel SAMCEF est utilisé. Celui permet, entre autre de rentrer une loi utilisateur entre 


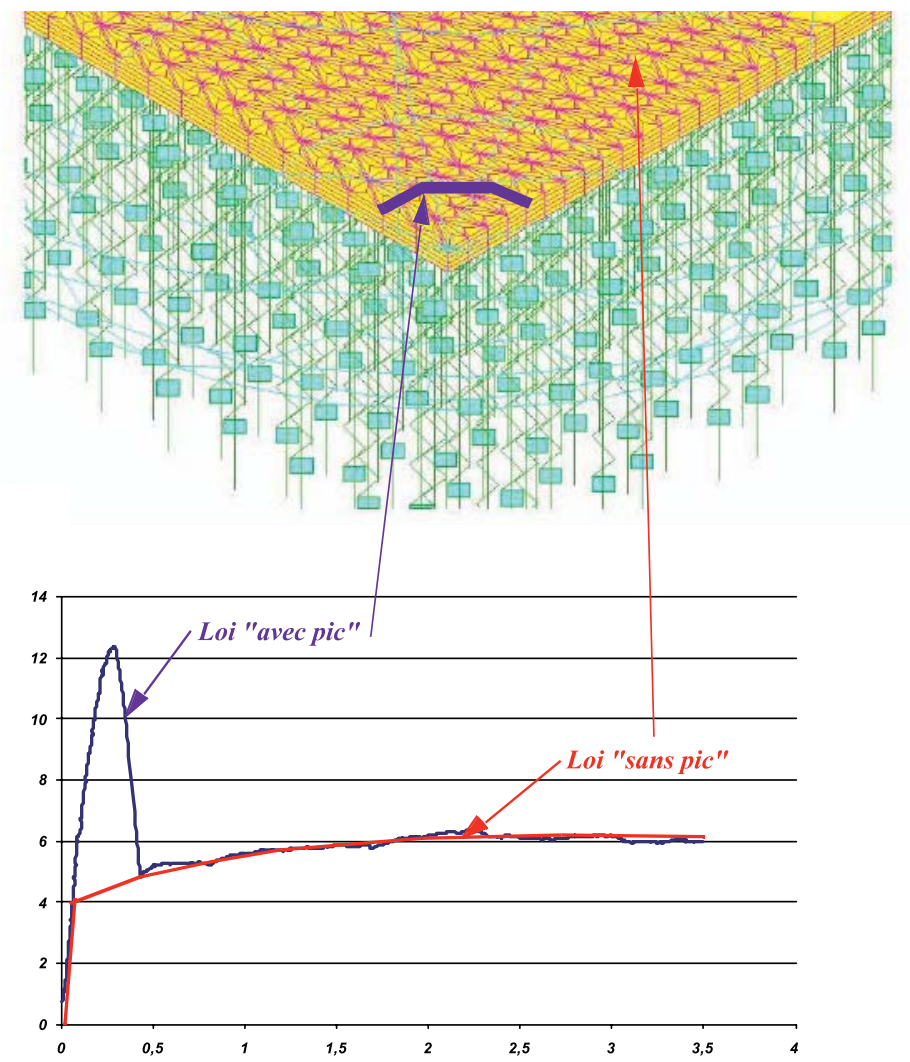

Fig. 20. Modélisation éléments finis du problème d'indentation sur sandwich à peaux métalliques épaisses.

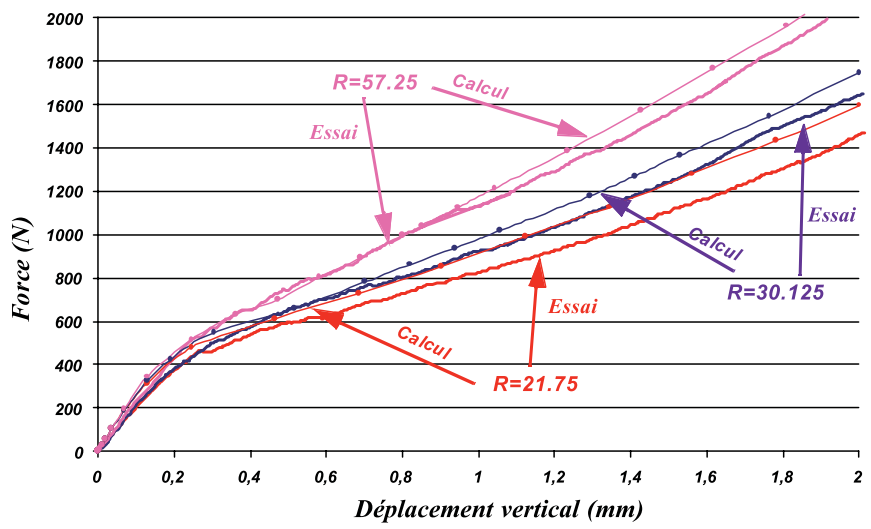

Fig. 21. Corrélation essai/modèle numérique dans le cas sandwich à peaux métalliques épaisses.

les degrés de liberté de l'élément [24]. Dans notre cas la loi non-linéaire d'écrasement évolue en fonction de la rotation aux nœuds de la peau entre une loi « avec pic » (cas $\theta=0)$ à une loi sans pic ( $\left.\operatorname{cas} \theta>\theta_{\text {Limite }}\right)$, voir figure 24 . La rotation $\theta$ utilisée est la moyenne quadratique des rotations suivant les deux axes locaux du plan tangent de la peau en flexion. L'angle $\theta_{\text {Limite }}$ est trouvé en dépouillant les rotations obtenues numériquement sur les arêtes de la première cellule. Après plusieurs tests numériques, on a fixé $\theta_{\text {Limite }}=2,3^{\circ}$. Différents tests numériques ont été effectuées sur la position du point de raccordement et la loi de décroissance entre $\theta$ et $\theta_{\text {Limite }}$ (décroissance linéaire

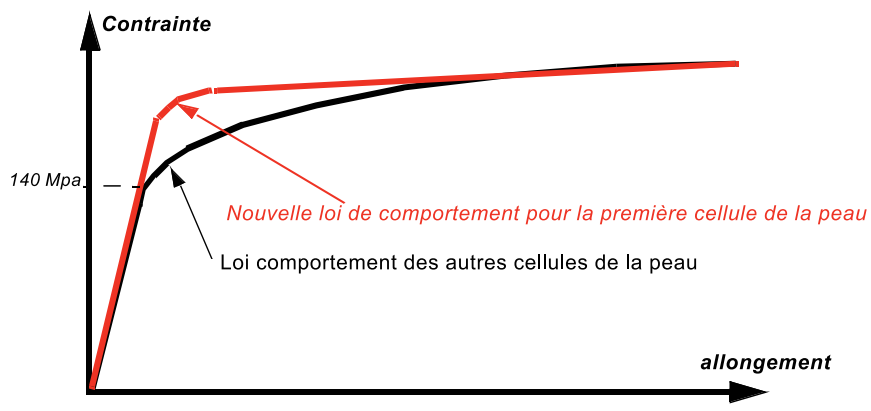

Fig. 22. Modèle unifié : modification de la loi de comportement de la première cellule de la peau.

ou parabolique). Globalement, ces tests ont montré une influence faible de ces deux paramètres. Une corrélation améliorée est obtenue à la fois pour les peaux fines et les peaux épaisses (Fig. 25).

\section{Conclusions}

Une modélisation de l'indentation des structures sandwichs avec peaux métalliques permettant une bonne corrélation des lois de contact a été développée. Une étude phénoménologique du plissement a permis de proposer une modélisation originale de l'âme en nid d'abeille Nomex ${ }^{\mathrm{TM}}$. Cette étude a permis de montrer que l'essentiel des efforts de compression était repris par les 

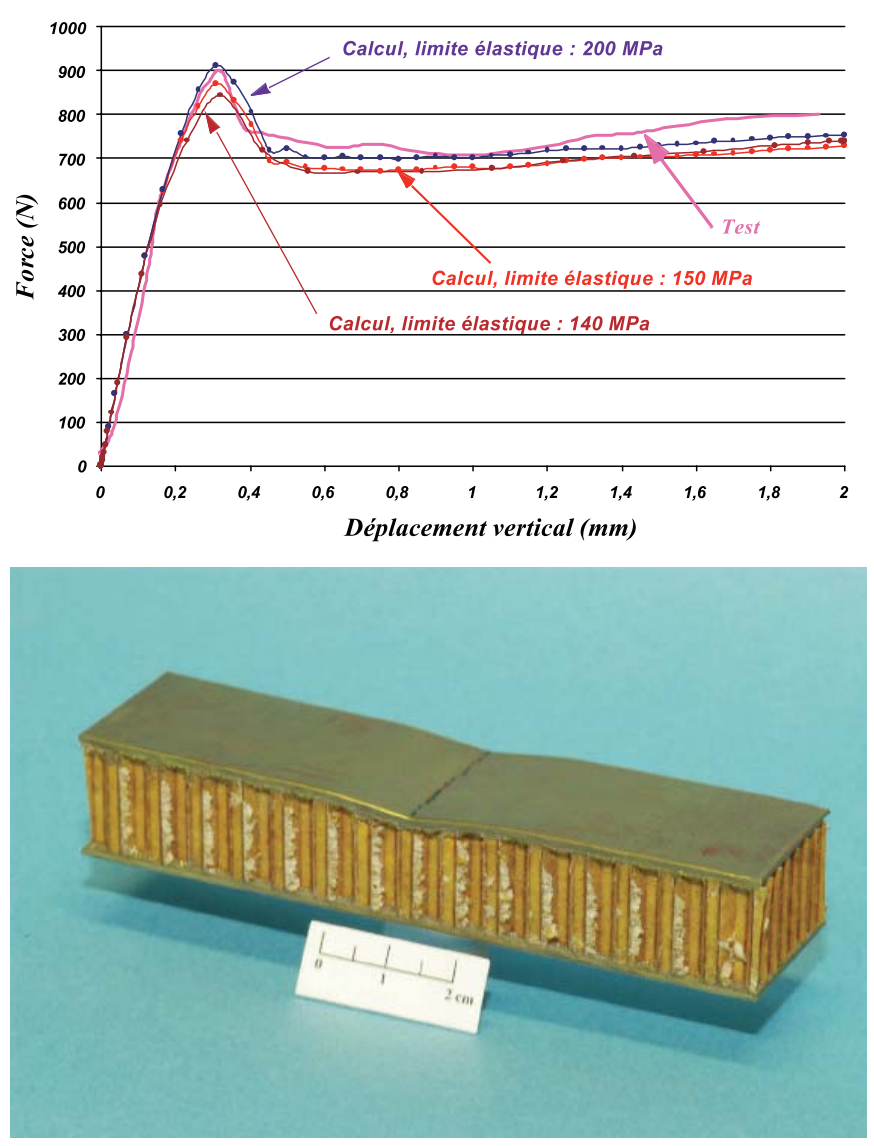

Fig. 23. Calibration de la limite élastique.

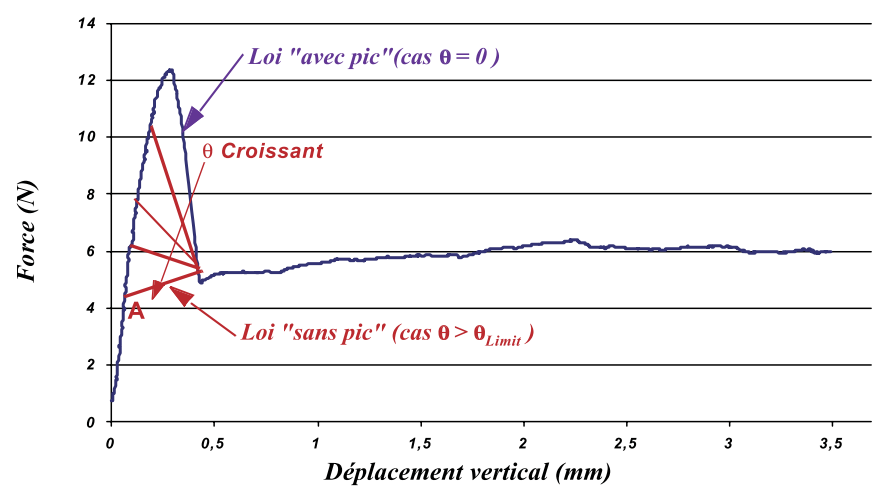

Fig. 24. Loi d'écrasement unifiée.

arêtes verticales de la structure hexagonale. Ainsi le nid d'abeille peut être représenté par une grille de ressorts non-linéaires dont la loi d'écrasement est obtenue par un essai de compression uniforme d'un pain de nida. Lorsque l'indentation sur nida seul doit être modélisée, une éprouvette avec le nida collé sur une seule peau en aluminium est utilisée. Du fait de conditions aux limites différentes, une loi d'écrasement obtenue avec des éprouvettes de nida collées avec deux peaux est utilisée pour modéliser l'indentation de structures sandwichs à peaux métalliques. L'utilisation directe de cette loi donne de bons résultats lorsque des peaux fines $(0,1 \mathrm{~mm})$ sont utilisées. Dans le cas de peaux plus épaisses, la raideur

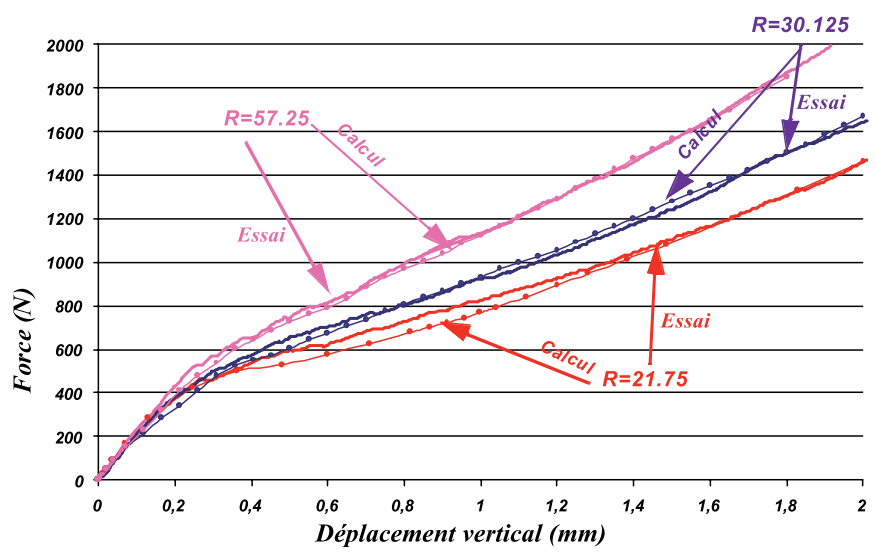

Fig. 25. Corrélation des essais d'indentation avec le modèle unifié (cas des peaux épaisses).

de flexion de la peau modifie la réponse des arêtes verticales du nida et la loi «avec pic » doit être corrigée progressivement jusqu'à une loi « sans pic». Cette sensibilité aux conditions aux limites a d'ailleurs été soulignée lors de l'étude phénoménologique. Par ailleurs, une forte triaxialité des contraintes dans la peau au droit de l'indenteur oblige à utiliser soit une modélisation volumique pour la peau, soit à augmenter artificiellement la limite élastique pour la zone de la peau correspondant à la première cellule de nida écrasé. Cette approche permet de corréler avec une très bonne précision les essais d'indentation sur support plat. Des développements ultérieurs ont par ailleurs permis de démontrer que cette approche était valide dans le cas d'essais de flexion 3 points et d'essais dynamiques. L'approche actuellement testée pour la modélisation de la compression après impact de sandwich à peaux métalliques. Enfin, dans un second temps, la simulation de l'impact sur des peaux composites en prenant en compte les dégradations de celles-ci pourra être envisagée. Il semble par ailleurs que l'approche qui a été développée soit utilisable pour d'autres âmes en matériaux cellulaires car le mécanisme de plissement est très commun. Une approche multi-niveau de l'évaluation des dommages d'impact est envisageable dans le cas de structures complexes ou de dommages multiples. Enfin, la relative simplicité du modèle unifié doit permettre à terme de l'inclure de manière aisée dans une boucle de reverse engineering permettant à partir d'une photo tridimensionnelle de l'impact, de déterminer après simulation du dommage, la résistance résiduelle de la structure.

\section{Références}

[1] S. Abrate, Localized impact on sandwich structure with laminated facings, Appl. Mech. Rev. 50 (1997) 69-82

[2] D. Guedra-Degeorges, P. Thevenet, S. Maison, Damage tolerance of sandwich structures, Proceedings of the Euromech 360 Colloquium, Saint-Étienne 1997, A. Vautrin, Kluwer Academic Publishers, 1997, pp. 29-36 
[3] H. Razi, B. Sergeev, S. Shkarayev, E. Madenci, Analysis of sandwich panels with multiple-site damage, Engineering Fracture Mechanics 64 (1999) 255-268

[4] R. Ferri, Sankar, Static indentation and low velocity impact tests on sandwich plates, Proceedings of the 1997 ASME International Mechanical Engineering Congress and Exposition, Dallas, TX, USA, 55 (1997) 485-490

[5] J. Williamson, P.A. Lagace, Response mechanism in the impact of graphite/epoxy honeycomb sandwich panels. Proceedings of the 8th technical conference of the american society for composites, Cleveland, OH, USA, Technomic Publishing Company, 1994, 287-297

[6] E.J. Herup, A.N. Palazotto, Low-velocity impact damage initiation in graphite/epoxy/nomex honeycomb-sandwich plates, Composite science and technology 57 (1997) 15811598

[7] R.S. Swanson, K. Jongman, Design of sandwich structures under contact loading, Composite Structures 59 (2003) 403-413

[8] P. Soden, Indentation of composite sandwich beams, J. Strain Analysis 31 (1996) 353-360

[9] R. Olsson, H.L. McManus, Simplified theory for contact indentation of sandwich panels, Proceedings of the AIAA/ASME/ASCE/AHS/ASC 36th Structural dynamic and material conference, New Orleans, LA, AIAA95-1374-CP, 1995, pp. 1812-1820

[10] M.L. Bernard, P.A. Lagace, Impact resistance of composite sandwich plates, J. Reinforced Plastics and Composites 8 (1989) 432-445

[11] T.K. Tsotsis, S.M. Lee, Characterization of localized failure modes in honeycomb sandwich panels using indentation, ASTM STP 1274, 1996, pp. 139-165

[12] R.K. McFarland Jr, Hexagonal cell structures under postbuckling load, AIAA J. 1 (1963) 1380-85

[13] T. Wierzbicki, Crushing analysis of metal honeycomb, Int. J. Impact Engineering 1 (1983) 157-174

[14] Enboa Wu, Wu-shung Jiang, Axial crush of metallic honeycomb, Int. J. Impact Engineering 19(5-6) (1997) 439-456
[15] Y. Aminanda, B. Castanié, J.J. Barrau, P. Thevenet, D. Guedra-Degeorges, Étude expérimentale et modélisation du comportement en compression des structures nid d'abeille, J. Phys. IV France 12 (2002) Pr11-219-Pr11226

[16] R.S. Thomson, A.P. Mouritz, Skin wrinkling of Impact Damaged Sandwich Composite, J. Sandwich Structures and Materials 1 (1999) 299-322

[17] T. Besant, G.A.O. Davies, D. Hitchings, Finite element modelling of low velocity impact of composite sandwich panels, Composite Part A 32 (2001) 1189-1196

[18] Qixuan Zheng, Zhengneng Li, Di Wu, Damage analysis of honeycomb core composite sandwich plate subjected low velocity impact, J. Reinforced Plastics and Composites 19 (2000) 58-68

[19] P.J. Minguet, A model for predicting the behavior of impact-damaged minimum gage sandwich panels under compression. Proceedings of the AIAA/ ASME/ASCE/AHS/ASC 32th Structural dynamic and material conference, Baltimore, MD, AIAA-91-1075-CP, 1991, pp. 1112-1122

[20] D.P.W. Horrigan, R.R. Aitken, G. Moltschaniwskyj, Modelling of crushing due to impact in honeycomb sandwiches, J. Sandwich Structures and Materials 2 (2000) $131-151$

[21] T.E. Lacy, Y. Hwang, Numerical modeling of impactdamaged sandwich composites subjected to compressionafter-impact loading, Composite Structures 61 (2003) $115-128$

[22] C.M. Kindervater, H. Georgi, Composite strength and energy absorption as an aspect of structural crash resistance, in Structural Crashworthiness and Failure edited by Norman Jones and Tomas Wierzbicki, Elsevier Applied Science, 1993

[23] A. Petras, M.P.F. Sutcliffe, Indentation Failure analysis for sandwich beams, Composite Structures 50 (2000) $311-318$

[24] SAMCEF user's manual V9.1, SAMTECH Group, Liège, Belgium

Retrouvez nos articles sur le site : www.edpsciences.org/meca 